\title{
Desarrollando las habilidades sociales desde la escuela como impulso de una cultura de paz.
}

\section{Gloria López ${ }^{1}$ y Yuherqui Guaimaro. ${ }^{2}$}

Artículo científico

Material original autorizado para su primera publicación en el Journal de Ciencias Sociales, Revista Académica de la Facultad de Ciencias Sociales de la Universidad de Palermo.

Recibido: 18-10-2013

Aceptado: 12-3- 2014

\section{Resumen:}

La escuela es el entorno social donde el niño se relaciona en gran parte de su tiempo con sus iguales y con adultos, lo que la convierte en una de las instituciones de socialización más relevantes, destinada a potenciar y enseñar las habilidades sociales. Esta necesidad, de abordar los estilos de convivencia escolar, surge al constatar que en los centros educativos se ha incrementado con niveles alarmantes los problemas de relación interpersonal entre los niños y las dificultades de interacción con y entre familias. La atención en los temas de investigación en el ámbito escolar se han centrado en los aspectos cognitivos, dejando al margen aspectos relevantes como lo son las necesidades evolutivas básicas de los niños y las niñas, caben mencionar: identidad personal, autoestima, seguridad emocional, relaciones sociales y redes, participación, desarrollo de autonomía, etc. (López, 1995). Basados en una educación en valores se pretende favorecer en la escuela un ambiente donde se construyan comportamientos y actitudes prosociales en sus actores contribuyendo de esta manera con otros sectores e instituciones de la sociedad a construir la cultura de paz.

En una sociedad cada vez más competitiva donde se penaliza o se recompensa al sujeto por las consecuencias de sus decisiones y tomando en cuenta que, además, el sujeto se encuentra inmerso en una cultura individualista donde se siente cada día más solo frente al resultado de las decisiones que toma, se hace necesario favorecer el desarrollo social en un entorno donde el sujeto y los grupos afronten sus conflictos y aprendan a manejar constructivamente sus diferencias. Si dotamos a los niños y niñas de una formación en valores que favorecen una adecuada convivencia y les otorgamos herramientas de negociación y mediación estaremos sentando las bases de una verdadera cultura de paz.

Palabras clave: habilidades sociales, cultura de paz, convivencia, educación en valores.

\begin{abstract}
:
The school is the social environment where the child relates in large part of their time with to their peers and adults, making it one of the most important institutions of socialization and designed to teach and enhance social skills. This need to address school lifestyles that emerge in schools has increased to alarming levels of interpersonal problems among children and the difficulties of interaction where the individual between families. The focus on research issues in schools has

1 Psicóloga Social Universidad Central de Venezuela. Directora Centro de Investigaciones para la Infancia y la Familia (CENDIF), UNIMET. glopez@unimet.edu.ve

2 Socióloga, Universidad Central de Venezuela. Investigador Asociado al Centro de Investigaciones para la Infancia y la Familia (CENDIF), UNIMET.
\end{abstract}


centered on cognitive aspects, leaving out important aspects such as basic developmental needs of children, for instance: personal identity, self-esteem, emotional security, relationships and social networking, participation, development of autonomy, and so on (Lopez, 1995). Based on an education oriented at values the school seeks to foster an environment where behavior and pro-social attitudes are built into the community contributing with other sectors and institutions of society to build a culture of peace. In an increasingly competitive society where the individual is penalized or rewarded for the consequences of their decisions and taking into account that, in addition, the person is immersed in an individualistic culture where he feels increasingly alone every day with the outcome of the decisions he makes, it is necessary to promote social development in an environment where the individual and the groups to address their conflicts and learn to deal with their differences constructively. If we equip children with an education in values that promote proper behavior and the tools for negotiation and mediation we will be laying the foundation of a true culture of peace.

Keywords: social skills, culture of peace, coexistence, values education.

\section{Introducción}

La Asamblea General de Naciones Unidas define la Cultura de Paz como "Un conjunto de valores, actitudes, tradiciones, comportamientos y estilos de vida", basados en una serie de derechos fundamentales para el desarrollo de una vida plena y pacífica, marcándonos unas metas a conseguir y unos valores a transmitir, los cuales se recogen en el Manifiesto 2000.

Desde esta perspectiva pensamos que sin duda es la escuela, a través de la educación, el instrumento óptimo para construirla. Nuestro compromiso como padres, maestros, profesionales, etc., es promover la paz como acción colectiva e individual, saber convivir con los conflictos y detener, disminuir y, en cualquier caso, prevenir las manifestaciones de violencia (Tuvilla, 2003).

A través de la proclamación por Naciones Unidas del decenio 2000-2010 como Decenio Internacional de una Cultura de Paz y No violencia para los niños del mundo se insta a los países a adquirir el compromiso de fomentar la Cultura de Paz en todos los ámbitos de la vida. El presente trabajo se contextualiza en esta declaración relevante en pro de los Derechos Humanos y de la Cultura de Paz.

Son muchos los países y organizaciones que se han sumado a este compromiso, desarrollando políticas educativas que vienen a fomentar la Cultura de Paz (Sánchez, 2007).

Educación para la paz es aquella que va “...dirigida hacia el pleno desarrollo de la personalidad humana y el fortalecimiento del respeto de los derechos humanos y las libertades fundamentales". Promueve "...la comprensión, la tolerancia y la amistad entre todas las naciones, grupos étnicos o religiosos" y fomenta "...las actividades de las Naciones Unidas para el mantenimiento de la paz" (artículo 26, Declaración Universal de los Derechos Humanos).

\section{Justificación}

La convivencia es uno de los aspectos básicos y relevantes en lo que respecta a las relaciones humanas. En toda relación surgen desavenencias que pueden dar lugar a conflictos interpersonales; 
por tanto, convivencia y conflicto se presentan en un mismo escenario. Los seres humanos vivimos en una sociedad donde los conflictos, a veces, se resuelven con violencia. Hoy nos alarma el incremento de hechos violentos protagonizados por niños y niñas, y así lo informan con relativa frecuencia los medios de comunicación (Funes, 1998; Rojas, 1996).

Entendemos la violencia como el ejercicio del poder mediante el empleo de la fuerza. Implica la existencia de un ser fuerte y uno débil donde el fuerte utiliza el poder de manera constante para abusar del vulnerable y tomar el control por la fuerza.

Existen diferentes tipos de violencia: la estructural, arraigada en las estructuras sociales y consiste en la predisposición de las condiciones de vida de las personas y las comunidades desde el punto de vista económico, laboral y social minimizando su posibilidad de elegir sobre sus condiciones de vida; la cultural, se refiere a aquellos aspectos culturales que terminan por legitimar el abuso de poder y la discriminación, tal es el caso de las creencias y las costumbres; y la violencia directa, la que se manifiesta a través del abuso, de manera intencional y repetitiva, cabe mencionar la violencia física y la psicológica.

Ante los hechos de violencia surge la cultura de paz, la que asegura una distribución más equitativa del poder y por lo tanto implica la influencia mutua y no el dominio de uno sobre otro.

La cultura de paz es un proceso de aprendizaje colectivo basado en los valores de justicia, responsabilidad, libertad, cooperación, respeto, solidaridad y tolerancia, orientado a incluir a todas las personas en la toma de decisiones para una distribución equitativa del poder.

Partiendo de la urgente necesidad de educar para la formación de ciudadanos/as cuyo objetivo sea la adecuada convivencia, entonces necesitamos una educación que promueva una cultura de convivencia incluyente y justa, por lo cual educar para la paz significa educar en valores y educar sin violencia en el compromiso colectivo y comunitario de promover la buena convivencia.

Las escuelas no escapan a este fenómeno, la conflictividad escolar se ha convertido en una de las mayores preocupaciones de la sociedad. A pesar de que la mayor parte de las prácticas educativas son favorecedoras de una buena convivencia, y que las relaciones interpersonales que se dan en este ámbito habitualmente son de cooperación, ayuda, estima, aprecio, etc., existen casos concretos sobre agresiones o acoso, denigración, discriminación a compañeros y compañeras, y son una de las principales preocupaciones de los docentes y de los padres y representantes, y por tanto, se hace cada vez más necesario aprender a ser más conscientes de la importancia de abordar el tema.

Es por ello que Cendif-Unimet enfoca los esfuerzos hacia la escuela con el objetivo de que los maestros/as, alumnos/as y madres/padres de familia asuman el reto de ser agentes multiplicadores de procesos educativos que transformen los conflictos en recursos y potencialidades creadoras de paz colectiva.

\section{Perspectiva teórica}

El tema de la competencia social en infancia y adolescencia ha recibido una marcada atención en los últimos años y este interés dio lugar a la constatación de la importancia de las habilidades sociales en el desarrollo infantil y en el posterior funcionamiento psicológico, académico y social. Son numerosas las investigaciones que señalan la relación existente entre competencia social y 
logros escolares, sociales y ajuste personal y social por el contrario la incompetencia social se relaciona con los desajustes y las dificultades, tales como: baja aceptación, rechazo, aislamiento, problemas emocionales y escolares, desajustes psicológicos y psicopatología infantil, delincuencia juvenil y diversos problemas de salud mental que se vivencian en la vida adulta (Elliot y Gresham, 1991; Hundert, 1995; Goldstein, Sprafkin, Gershaw y Klein, 1989; Michelson, Sugai, Wood y Kazdin, 1987; Pelechano, 1996).

Las situaciones conflictivas deben considerarse y resolverse de manera no violenta. Son varios los autores que apoyan y defienden esta visión positiva, podemos citar a Galtung (2003) quien aboga por una perspectiva positiva del conflicto, viéndolo como una fuerza motivadora de cambio personal y social.

Para Lederach (2000), el conflicto es una paradoja, porque supone una interacción entre dos adversarios que compiten por sus intereses, pero que a la vez han de cooperar para llegar a acuerdos, esta interdependencia nos hace ver el conflicto como primordial y necesario para el crecimiento de la persona.

Por su parte los conflictos son el motor y la expresión de las relaciones humanas (Vinyamata, 2005). El conflicto es, signo de diversidad; cada persona tiene sus propias opiniones, vivencias, emociones, que no siempre se corresponden con las de los demás, y es la forma de enfrentarse a él la que va a determinar su transformación. Desde esta perspectiva, el conflicto se nos presenta como una ocasión de aprendizaje en la construcción de nuestras relaciones y un motor de cambio, por tanto, debemos entenderlo no sólo como natural, sino como positivo en toda sociedad democrática.

Y Binaburo (2007) nos agrega en lo que respecta a educación debemos abogar por "educar desde el conflicto" como recurso de aprendizaje.

Se diferencian dos técnicas y estrategias de resolución de conflictos, las que son preventivas que evitan su surgimiento y las de resolución que ofrecen alternativas para solventarlo. Una de las actividades primordiales del docente es precisamente hacer que los escolares aprendan a aclarar sus conflictos en el aula, previniendo así situaciones de violencia. Es lo que Goleman (1997) llama "La escolarización de las emociones", donde profesores y estudiantes incorporan situaciones del acontecer diario, compartiendo realidades como sentirse ofendido, rechazado, envidiado, etc., que sirven de reflexión y análisis para la búsqueda de soluciones positivas.

Al parecer las políticas preventivas y las líneas de intervención que se han seguido en los centros educativos, no han logrando detener el avance de los comportamientos violentos, los resultados son insuficientes y aún el problema persiste con alta incidencia en la frecuencia y en las consecuencias cada vez revestidas de mayor gravedad.

\section{Habilidades Sociales}

Para Monjas (1999) las habilidades sociales son “... conductas necesarias para interactuar y relacionarse con los iguales y con los adultos de forma efectiva y mutuamente satisfactoria". Pero al revisar la bibliografía encontramos una gran variedad de términos, tales como: habilidades sociales, 
habilidades de interacción social, habilidades interpersonales, destrezas sociales, habilidades de intercambio social, conducta interactiva, relaciones interpersonales, etc. (Caballo, 1993; Elliot y Gresham, 1991; Hundert, 1995).

Según Gresham $(1986,1988)$ se suelen encontrar tres tipos de definiciones de las habilidades sociales: i) definición de aceptación de los iguales. En estas definiciones se usan índices de aceptación de los iguales o popularidad. Se consideran niños socialmente hábiles los que son aceptados o populares en la escuela o en la comunidad. El mayor fallo de estas definiciones es que no identifican los comportamientos específicos que se relacionan con la aceptación de los iguales; ii) definición conductual, donde se definen las habilidades sociales como aquellos comportamientos específicos de la situación que maximizan la probabilidad de asegurar o mantener el reforzamiento o decrecer la probabilidad de castigo o extinción contingente sobre el comportamiento social propio. La premisa que subyace a este tipo de definiciones es la adquisición de habilidades interpersonales específicas que permiten a uno experimentar relaciones con otros que son personal o mutuamente satisfactorias. Estas definiciones tienen la ventaja de que se pueden identificar, especificar y operacionalizar los antecedentes y las consecuencias de los comportamientos sociales particulares con fines de evaluación e intervención. Sin embargo, esta definición no asegura que los comportamientos identificados para la intervención sean de hecho socialmente hábiles o socialmente importantes; y iii) definición de validación social en donde las habilidades sociales son aquellos comportamientos que, en situaciones determinadas, predicen importantes resultados sociales para el niño, como por ejemplo, aceptación, popularidad o juicios de otros significativos.

Basados en estas premisas, entenderemos por habilidades sociales al conjunto de competencias conductuales que posibilitan que el niño, niña o adolescente mantenga relaciones sociales positivas con los otros y que afronte, de modo efectivo y adaptativo, las demandas de su entorno social, aspectos estos que contribuyen significativamente, por una parte, a la aceptación por los compañeros y, por otra, a su adecuado ajuste y adaptación social, logrando superar y resolver de manera adecuada las situaciones de conflicto y manteniendo una adecuada convivencia. Entonces, al hablar de habilidades sociales, nos referiremos a un conjunto de conductas aprendidas que, además, se revelan de manera adecuada o aceptadas socialmente, como ejemplo, caben mencionar: hacer una solicitud, responder al saludo, ponerte en el lugar del otro, decir cosas agradables y positivas a los que te rodean.

Las investigaciones realizadas demuestran que el mejor entrenamiento en habilidades sociales es efectivo en la enseñanza de conductas socialmente hábiles a niños, niñas y adolescentes. En la actualidad se dispone de suficiente evidencia sobre técnicas, estrategias y procedimientos que se pueden utilizar para la enseñanza de conductas de interacción social en la infancia (Beck y Forehand, 1984; Caballo, 1993; Gresham, 1988; Gresham y Lemanek, 1983; Ladd y Asher, 1985, entre otros).

Otras investigaciones ponen de manifiesto que la interacción entre alumnos, especialmente la de tipo cooperativo, favorece la adquisición de competencias y destrezas sociales y el rendimiento escolar (Echeita y Martín, 1990; Trianes, 1996; Trianes, Jiménez y Muñoz, 1997). Además, hay que tomar en cuenta que la educación es un proceso de interacción comunicativa entre niños/as, entre maestros/as, entre padres y a su vez niños/maestros, niños/padres y padres/maestros. De allí que la educación puede considerarse como una actividad que no puede darse al margen de unas 
relaciones interpersonales estructuradas, y es a esto a lo que Báez y Jiménez (1994) denominaron interacción educativa.

Por su parte, Goleman (1997) y Goñi (1996) agregan que en el contexto escolar además de las relaciones interpersonales, también se ignoran otros aspectos básicos del desarrollo de la personalidad integral del niño, niña y adolescente, tales como: las emociones y los afectos; la autoestima y el autoconcepto; la competencia personal y social; y la inteligencia emocional.

\section{Adquisición y desarrollo de las habilidades sociales}

Según López (1995) el desarrollo social se entiende desde un modelo explicativo interactivo en el que biología y cultura son factores activos que se influyen mutuamente y hacen del resultado siempre una versión individual y distinta de la persona humana. Entre las necesidades del ser humano está la de construir vínculos afectivos y sociales para sentirse seguro y acompañado en lugar de solo y abandonado, ya que es un ser social que sólo resuelve sus necesidades básicas en la relación con los demás (López y Fuentes, 1994).

La familia y las primeras figuras de apego desempeñan, sobre todo en los años iniciales del niño y de la niña, un papel muy relevante en este proceso de desarrollo de la competencia interpersonal (Echeburúa, 1993), y agrega este autor que "...la estimulación social que hacen los padres (por ejemplo, relaciones con vecinos, juegos colectivos, etc...) correlaciona directamente con el grado de desenvoltura social de los niños y las niñas".

De allí que, se facilita la adquisición de habilidades sociales y se disipan los temores sociales iniciales cuando los padres facilitan la exposición a situaciones sociales nuevas y diversas, y por el contrario, se fortalece en los niños y las niñas la inhibición, la timidez y la poca sociabilidad cuando los padres evitan exponerlos a situaciones sociales. Los niños aprenden un conjunto de habilidades sociales que pueden considerarse muy escasos manifestando conductas de inhibición y de evitación (Monjas, 2000).

Además de la familia los niños y las niñas interaccionan con otras figuras significativas, y esto sucede en el momento en que ingresan al sistema escolar, caben mencionar: sus pares o iguales, compañeros y compañeras mayores y menores a su edad, y los maestros y maestras.

En resumen, podemos afirmar que la competencia social se desarrolla y se aprende a lo largo del proceso de socialización, gracias a la interacción con otras personas y propiciada básicamente por los diferentes mecanismos del aprendizaje. Estos son: i) la experiencia directa, que corresponde al aprendizaje en función de las respuestas de aversión o aceptación obtenidas por su comportamiento; ii) la observación, que hace referencia al aprendizaje de conductas de relación como resultado de la exposición ante modelos significativos; iii) el aprendizaje instruccional, que tiene que ver con el aprender a través de lo que se le expresa verbalmente directa o sistemáticamente; y por último, iv) el aprendizaje por reforzamiento social o por ausencia del mismo, vivenciado en la interacción con otros o por los observadores del entorno. 


\section{Contexto Venezolano}

El Ministerio del Poder Popular Para Relaciones Interiores, Justicia y Paz (Min-Justicia) ha estado realizando talleres de Prevención de la Violencia Escolar en todo el país, en el contexto de la Gran Misión A Toda Vida Venezuela en su primer vértice.

Para combatir la violencia en el ámbito escolar no solo debemos preocuparnos por instruir a los niños, también se debe proporcionar a los padres, a los maestros y a los directivos docentes y administrativos, las herramientas que les faciliten la identificación de las agresiones y cómo actuar en consecuencia; ya que diversas investigaciones demuestran que en las relaciones con estos y sus prácticas, deben ser considerados en general en el proceso educativo, y en particular, en el tema de la convivencia. En los recintos escolares se pueden encontrar varios tipos de violencia, se destacan: las descalificaciones, el lenguaje obsceno, los golpes, el hurto y los ataques sexuales.

Entre los factores de riesgo que influyen en la aparición del fenómeno caben mencionar, la exclusión social, la naturalización de la violencia en la sociedad, la exposición continuada a hechos violentos, especialmente en los medios de comunicación, la carencia de formación en valores y, además, el escaso manejo de herramientas para la resolución de conflictos. Se destaca el ausentismo escolar como una de las principales consecuencias del fenómeno.

Por otra parte, el agresor presenta conductas de liderazgo y cuenta con personas que apoyan su comportamiento violento. Estas personas presentan baja tolerancia a la frustración y muestran dificultades para relacionarse con personas de autoridad, al igual que para cumplir normas y respetar los límites.

Se ha incrementado en un $70 \%$ el índice de violencia escolar en los planteles educativos, según estadísticas que maneja el Consejo Municipal de Derecho del Niño, Niña y Adolescente. La profesora Miriam Álvarez (Diario La Voz, 2013), titular de esa dependencia del gobierno local, dijo que las autoridades locales están preocupadas por esta problemática, por lo que se han unido al Programa Nacional denominado Movimiento por la Paz. "Este plan de trabajo comenzó a principios de año de forma individual y limitado, pero a partir del mes de abril se ha estado llevando a los planteles a petición de los docentes", indicó la funcionaria.

Invedin y la Fundación Cadena Capriles, organizaciones preocupadas por el incremento de los hechos de violencia escolar en el país se han dado a la tarea de dictar charlas educativas en el tema. Con esta actividad, ambas organizaciones han buscado profundizar en el tema de la violencia en las escuelas, asunto que se ha convertido en un gran problema para todos los miembros de la comunidad educativa, en especial a los docentes y representantes.

El coordinador de la Fundación Cadena Capriles (Diario Últimas Noticias, 2013), explicó que desde 2004, luego de visitar centros de estudios en varios lugares de Caracas y de Venezuela, donde impartieron diferentes talleres, detectaron la "...preocupación de la comunidad educativa, así como de padres y representantes en el tema de la violencia escolar", por ello, a parte de los cursos, se plantean organizar foros donde las comunidades, las escuelas y los padres conozcan este problema y cómo confrontarlo.

La presidenta de Invedin (Diario Últimas Noticias, 2013), considera que los estudiantes deben manejar las herramientas, las escuelas deben estar en buenas condiciones, no hayan lugares que sea 
escondrijos para acciones inaceptables, la dirección de la escuela conozca bien el espacio escolar y tome medidas de los lugares solitarios, deben promover actividades que mejoren las relaciones, los maestros deben reflexionar y ver el problema no "...de forma externa sino como parte de su entorno y cómo los ha afectado, para que estos casos los manejen mejor, porque se han detectado casos de violencia de maestros a niños".

Algunas cifras de la violencia en las escuelas (Diario Últimas Noticias, 2011) reportan que el $63 \%$ de los profesores de educación media han presenciado actos violentos en los planteles educativos. La Fiscalía $N^{\circ} 18$ (con competencia en responsabilidad penal del adolescente) reporta 70 agresiones al mes en las escuelas. Según Cecodap un $88 \%$ del personal de los planteles y estudiantes entre $7^{\circ}$ y $9^{\circ}$ grado (Sector Catia y Petare, Caracas) reportaron haber sufrido agresión verbal, el $79 \%$ fue agredido físicamente, un $24 \%$ de los estudiantes consultados pide más vigilancia como solución al problema. La Federación Venezolana de Maestros de enero a mayo de 2011 recibió 47 denuncias de profesores agredidos o amenazados de muerte por alumnos.

A pesar de las estadísticas nacionales disponibles es difícil comprender la magnitud del tema, lo que si queda claro es que se requieren acciones inmediatas y fundamentadas.

\section{Contexto legal}

La LOPNNA (Ley Orgánica de Protección de Niños, Niñas y Adolescentes, 2007) en su artículo 270 aduce que si los casos de violencia escolar llegan hasta instancias de los consejos de protección del niño, niña y adolescentes estos pueden, según el caso, emitir órdenes de detención a aquellos padres que se nieguen a atender los problemas que enfrentan sus hijos victimarios. El documento legal dispone de una serie de artículos recomendaciones y sanciones contra la violencia escolar. Hay muchas medidas que la ley contempla y se pueden sugerir e implementar acciones para que el niño(a) o adolescente sea atendido basado en los artículos que están contemplados en la Lopnna. Cuando los casos pasan de lo verbal a lo físico entran un conjunto de organismos y entes contemplados en la ley como la Fiscalía y los tribunales especiales para adolescentes (12 y menos de 18 años) que atienden estos casos.

En el año 2009 se crea la Ley de Prohibición de Videojuegos y Juguetes bélicos que busca prohibir la fabricación, importación, distribución, compra, venta, alquiler y uso de videojuegos y juguetes bélicos. A pesar de su implementación, los niños, niñas y adolescentes siguen expuestos a situaciones violentas. De igual manera, no se han emprendido suficientes acciones y políticas reales y efectivas que garanticen un entorno sin violencia para niños, niñas y adolescentes.

La Ley de Responsabilidad Social en Radio y Televisión en gran parte se enfoca en proteger a la niñez y adolescencia, de contenidos inadecuados que puedan afectar su desarrollo integral, sin embargo en la actualidad existen programas en horarios donde el mayor porcentaje de audiencia son niños, niñas y adolescentes en los que se muestran contenidos no adecuados para ellos. Frente a esto sigue existiendo una carencia de políticas y acciones por parte del Estado que contribuyan con el cumplimiento de la Ley.

No existe una política de uso del tiempo libre y de recreación para los niños y jóvenes en el país. Según Fe y Alegría, con 160 centros educativos repartidos en toda Venezuela, el uso inadecuado del tiempo libre es un agente propiciador del maltrato, del consumo de sustancias estupefacientes 
y psicotrópicas, y del embarazo adolescente. También CECODAP afirma con respecto a esto, que la violencia intrafamiliar aumenta en los fines de semana y en las vacaciones, períodos en los que los niños, niñas y adolescentes se encuentran libres. En estos períodos sólo existen como opción recreativa los planes vacacionales privados, los cuales implican altos costos para las familias, lo que excluye a los sectores populares de actividades recreativas, lúdicas y de esparcimiento para estos períodos de tiempo.

El 95\% de los casos atendidos por jueces de Paz son hechos de violencia intrafamiliar. Aun cuando se carece de datos estadísticos comparables para el ámbito nacional y otros estudios que cuantifiquen y detecten el problema en toda su amplitud, los reportes de instituciones como por ejemplo, los cuerpos policiales, sector salud y justicia, revelan cifras muy altas en la incidencia de la violencia intrafamiliar que deben ser objeto de consideraciones con carácter prioritario en las políticas públicas. La violencia doméstica, el abuso y la explotación sexual son problemas que reflejan las inequidades asociadas al género e inciden de manera significativa en la salud sexual y reproductiva de adolescentes.

Sólo el $5 \%$ de los maltratos familiares son denunciados, es decir, sólo se denuncia el maltrato cuando este es brutal o muy reiterado. Muchas de las víctimas retiran las denuncias antes de los juicios, casi siempre por miedo y bajo amenazas. Un factor que hay que tomarse en cuenta es la dificultad que tiene la víctima para probar los hechos, como llevar testigos. Los certificados de lesiones aportados no siempre tienen el poder probatorio de la existencia de la violencia familiar, ya que si bien certifican las lesiones, no acreditan quién las produce.

Es importante destacar que el fenómeno de "naturalización de la violencia" ha propiciado que muchas de las víctimas no consideren las situaciones como denunciables ya sea porque consideran que los hechos de violencia no son suficientemente significativos o se sientan en minusvalía y desasistencia frente a órganos del Estado, caracterizados por la extrema burocratización para tramitar estos casos y ofrecer respuestas efectivas.

\section{¿Qué proponemos?}

El objetivo central es minimizar la violencia escolar en las escuelas a través del desarrollo de las habilidades sociales con estrategias y recursos lúdico-cooperativos. Por ello proponemos facilitar un proceso formativo y participativo basado en el desarrollo de las habilidades sociales, involucrando a los alumnos/as, maestros/as y madres-padres de familia en el diseño, implementación y evaluación de un plan de acción que permita desactivar la violencia y elevar la convivencia en la escuela a través del desarrollo de habilidades sociales.

A través de las relaciones interpersonales el individuo obtiene importantes refuerzos sociales del entorno más inmediato que favorecen su desarrollo integral y por ende, su calidad de vida.

Sin duda alguna existen muchos senderos educativos que conducen a la formación de una cultura de paz. En Cendif-Unimet hemos elegido la metodología que aporta la educación basada en una metodología crítica, participativa, dialogal y democrática.

Es una metodología dialéctica, donde se logra el proceso investigación-acción-participación. Por lo tanto, no hay metodología más congruente para trabajar la violencia que la que promueve la no violencia y termina por empoderar a sus actores. Además la metodología propuesta en educación 
para la paz y la no violencia está basada en varios planteamientos sociales, psicológicos y políticos que enfatizan en el trabajo horizontal y grupal. Un trabajo grupal centrado en lo participativo, reflexivo y vivencial. Evoluciona a través de una metodología participativa que implica revelar, desarrollar y generar recursos desde las propias necesidades y convivencia de los actores sociales para la toma de conciencia de los problemas de su entorno y la identificación de sus recursos, individuales y colectivos, que les permitan resolverlos.

Esto implica desarrollar una serie de habilidades y estrategias que conlleve a la construcción de grupo en un ambiente de aprecio y confianza, que favorezca la comunicación y la toma de decisiones para trabajar la cooperación. Trabajar en grupo no significa perder el propio estilo y la individualidad que acompaña la participación personal en una tarea común; es la única vía de aprender a fondo los beneficios de la convivencia pacífica y democrática. Se construye el diálogo sin olvidar que el pensamiento es individual, se ejercita la cooperación sin menoscabo de la responsabilidad individual y entendiendo la importancia del esfuerzo individual como aporte del éxito compartido. Para la resolución no violenta de conflictos se requiere de formas creativas y solidarias que favorezcan ambientes donde se preserven los valores del consenso, la negociación y el diálogo respetuoso.

\section{Pasos para generar una adecuada convivencia}

Acosta (2006) plantea que los pilares en los que se fundamenta una buena convivencia son: la cohesión del grupo con el que se quiere realizar cualquier actuación, la gestión democrática de normas, el trabajo positivo sobre regulación de los conflictos, la educación emocional y la educación en valores. El resultado del afianzamiento de todos estos aspectos dará lugar a un buen desarrollo de las habilidades socioemocionales.

Se busca estimular el trabajo grupal y la participación activa a través de la vivencia de un proceso que exige cumplir con cuatro etapas consecutivas. Estas son: diagnóstico participativo, asimilación, fortalecimiento y evaluación-seguimiento.

1. Diagnóstico participativo: implica la validación y el reconocimiento de las necesidades y estilos de convivencia de los actores sociales involucrados en el proceso, la idea básica es conocer el entorno de la comunidad o comunidades donde se encuentran ubicadas las escuelas.

2. Asimilación: implica un proceso formativo con el sistema de taller, que busca estimular el trabajo grupal y la participación activa, entendido como un proceso activo de transformación recíproca entre el facilitador y el participante basado en el desarrollo de las habilidades sociales para minimizar la violencia. Cabe mencionar entre los talleres básicos:

- Desarrollando habilidades sociales para la convivencia pacífica.

- Resolución pacífica de conflictos.

- Educación en valores.

- Promoviendo una cultura de paz.

- Actividades lúdicas para la paz en el trabajo con los niños y las niñas.

3. Fortalecimiento: la cual comprende la práctica de las habilidades sociales, desarrolladas en los talleres, promoviendo y motivando actividades de convivencia cooperativa que integren a los involucrados y generen procesos de mejora de su propia convivencia. 
3.1. Recreos cooperativos: promover, durante los momentos de esparcimiento escolar, juegos y actividades cooperativas para la vivencia de las habilidades sociales asimiladas en los talleres, ya que los juegos cooperativos ofrecen un recurso para desarrollar las habilidades sociales de un modo significativo.

3.2. Campañas para promover la importancia del juego a fin de posicionar dentro de las escuelas las actividades lúdicas como un derecho y un medio de socialización para favorecer y motivar la acción colaborativa y la buena convivencia. La actividad lúdica es la posibilidad de acción del niño y la niña, y por lo tanto su medio de expresión para construir conocimiento y modificar la realidad mediante la representación de un juego.

3.3. El rincón de la paz: promover y motivar la ubicación de un espacio físico dentro de la escuela con recursos lúdicos (juguetes, juegos y materiales didácticos), carteleras informativas, afiches, dibujos, etc., para propiciar e incentivar la cultura de paz, a través de la convivencia.

3.4. Promotores y promotoras de paz: favorecer la participación de los niños y las niñas, con apoyo de los maestros/as y de los padres y madres, en acciones de mejora de la convivencia no violenta. Permite contar con promotores y promotoras de paz con habilidades sociales para mejorar la convivencia desde los propios recursos comunitarios.

4. Evaluación y seguimiento: esta etapa esencial permite determinar el progreso de las actividades y tomar las medidas necesarias para resolver problemas, haciendo los ajustes necesarios en los objetivos y actividades.

\section{Al culminar las 4 etapas, tendremos:}

1. Participantes entrenados en habilidades sociales para la convivencia no violenta, estos serán promotores y promotoras capacitados en la disminución de la violencia escolar.

2. Construcción de una convivencia armónica basado en valores de justicia, libertad, tolerancia, solidaridad, responsabilidad cooperación y respeto.

3. Disminución de los casos de conducta violenta en la escuela.

4. Incremento del manejo asertivo de conflictos.

5. Fortalecimiento de la expresión positiva de las emociones. Los escolares aprenden a identificar y auto regular sus emociones.

6. Espacio físico designado a los recursos lúdicos.

7. Desarrollo de estrategia replicable en poblaciones similares.

8. Las inversiones durante este período de la vida no solo benefician de manera directa, sino que sus resultados se verán en el largo plazo beneficiando a la descendencia de esta población, haciendo de estas inversiones auto-sostenibles en el largo plazo y de máximo impacto.

\section{Reflexiones finales}

Las habilidades sociales no se adquieren si no se llevan a cabo actividades educativas de forma deliberada, o sea que no mejoran por la simple instrucción informal, se necesita una 
intervención directa, deliberada y sistemática.

Los niños pasan gran parte de su tiempo en la escuela relacionándose con sus pares y con los adultos y por ello el medio académico se constituye en la institución de socialización que proporciona un entorno favorable para potenciar y enseñar habilidades sociales a los actores involucrados en el proceso.

Se hace necesario ofrecer al niño la oportunidad para que se relacione espontáneamente con los demás compañeros, modificar la ambientación del aula y la ubicación del grupo para que cada niño de la clase tenga oportunidad de relacionarse con todos y cada uno de sus compañeros estableciendo sistemas de trabajo grupal y desarrollando juegos cooperativos.

Es básico establecer un trabajo coordinado y compartido con las familias, aprender a ser ciudadana y ciudadano es aprender a participar y convivir juntos bajo normas comunes para la construcción de una verdadera democracia. De allí que la participación de los padres y representantes en el proceso formativo es determinante.

Es competencia y responsabilidad de las instituciones educativas la enseñanza de las habilidades sociales en coordinación con la familia. Por lo que es conveniente incluir adecuadamente a los padres y formar a los maestros/as en el campo de las habilidades de interacción social a nivel profesional y personal.

Estos aspectos pueden deducirse de la lectura de los trabajos de los últimos años de diversos autores entre los que cabe mencionar a Goñi, 1996; Trianes, 1996; Trianes, Jiménez y Muñoz, 1997. Esto nos lleva a concluir y a afirmar que existe una creciente necesidad de incluir programas de enseñanza de las habilidades sociales dentro de la currícula de los institutos educativos del área inicial, primaria y secundaria.

Por eso, al hablar de la violencia escolar no debemos dejar de lado al docente y tomar en cuenta solo al niño. La experiencia cotidiana nos hace saber que en las escuelas existen relaciones de poder, que en muchos casos sigue privilegiando el modelo pedagógico tradicional y que son elementos que tienen que ver con la dinámica institucional y que pueden incidir para que la violencia se potencie o para que se produzcan cosas que hagan lugar a la violencia. Las relaciones existentes dentro de la institución serán las que favorezcan o desalienten la existencia de violencia.

La escuela sanciona al niño que no actúa de acuerdo a lo que la institución espera de él. Nuestro desafío es buscar el camino para lograr que el niño permanezca en el sistema educativo, mostrándole alternativas de relación diferentes a la violencia. Debemos repensar una respuesta pedagógica, en la cual sin perder lo normativo se articulen las necesidades de los niños. Lo importante es no descontextualizar al niño, él trae al entorno educativo los aprendizajes previos adquiridos en el proceso de socialización primaria; en su familia existen pautas y formas de actuar violentas o no violentas desde el punto de vista de los padres, que el docente irá conociendo en su interacción con el niño y que son parte de él.

Solo conociendo y entendiendo los diferentes entornos en los que el niño se desenvuelve podremos buscar el modo de evitar que el niño entre en conflicto; el conocer sus necesidades e intereses nos permitirá modificar poco a poco la situación y permitir que ocurran nuevos aprendizajes paulatinamente. 


\section{Referencias bibliográficas}

Acosta, A. (2006). Gestión de la convivencia y regulación pacífica de los conflictos en centros escolares, Orión. Revista técnico-profesional de la asociación de Orientadores de Secundaria de Granada, 3, 37-49.

Báez, B., y Jiménez, J. E. (1994). Contexto escolar y comportamiento social. En M. J. Rodrigo (Ed.). Contexto y desarrollo social. (pp. 189-222). Madrid: Síntesis.

Beck, S., y Forehand, R. (1984). Social skills training for children: A methodological and clinical review of behavior modification studies. Behavioural Psychoterapy, 12, 17-45.

Binaburo, J. y Muñoz, B. (2007). Educar desde el conflicto, Consejería de Educación de la Junta de Andalucía.

Caballo, V. E. (1993). Manual de evaluación y entrenamiento de las habilidades sociales. Madrid: Siglo Veintiuno.

Diario La voz (2013) http://www.diariolavoz.net/2013/05/26/se-incrementa-la-violenciaescolar-en-simonbolivar/

Diario Últimas Noticias (2011)

http://www.ultimasnoticias.com.ve/noticias/ciudad/educacion/cifras-clave-de-la-violencia en-lasescuelas.aspx

Diario Últimas Noticias (2013)

http://www.ultimasnoticias.com.ve/noticias/ciudad/educacion/la-lopna-es-estricta-con-el-temade-la-violencia-e.aspx

Echeita, G., y Martin. E. (1990). Interacción social y aprendizaje. En A. Marchesi, C. Coll, y J. Palacios (Com.). Desarrollo psicológico y educación III. Necesidades educativas especiales y aprendizaje escolar. Madrid: Alianza.

Echeburúa, E. (1993). Fobia social. Barcelona: Martínez Roca.

Elliot, S. N., y Gresham, F. M. (1991). Social skills intervention guide. Austin, TX: Pro-ed.

Funes, J. (1998). Sobre las nuevas formas de la violencia juvenil, Revista Comunicar 10, p. 98.

Galtung, J. (2003). Paz por medios pacíficos: paz y conflicto, desarrollo y civilización, Bilbao, Bakeaz. Goleman, D. (1997). Inteligencia emocional. Barcelona: Kairós.

Goldstein, A. P., Sprafkin, R. P., Gershaw, N. J., y Klein, P. (1989). Habilidades sociales y autocontrol en la adolescencia. Barcelona: Martinez Roca.

Goñi, A. (Ed.). (1996). Psicología de la educación sociopersonal. Madrid: Fundamentos.

Gresham, F. M. (1986). Conceptual issues in the assessment of social competence in children. En P. S. Strain, M. J. Guralnik, y H. M.Walker (Eds.). Children's social behavior. Development, assessment and modification. (pp. 143-179). Orlando, FL: Academic.

Gresham, F. M. (1988). Social skills: Conceptual and applied aspects of assessment, training and social validation. En J. C. Witt, S.N. Elliot, y F.M. Gresham (Eds.). Handbook of Behavior Therapy in Education. (pp. 523-546). New York: Plenum Press.

Gresham, F. M., y Lemanek, K. L. (1983). Social Skills: A review of cognitive- behavioral training procedures with children. Journal of Applied Developmental Psychology, 4, 239-261.

Hundert, J. (1995). Enhancing social competence in young students. Austin, TX: Pro-ed. 
Ladd,G. L., y Asher, S. R. (1985). Social skills training and children's peer relations. En L'Abate, y Milan (Eds.). Handbook of Social Skills Training and Research. (pp. 219-244). New York: John Wiley and Sons.

Lederach, J. (2000). El abecé de la paz y los conflictos, Los libros de La Catarata.

López, F. (1995). Necesidades de la infancia y protección infantil. Fundamentación teórica, clasificación y criterios educativos. Madrid: Ministerio de Asuntos Sociales.

López, F., y Fuentes, M. J. (1994). Revisión, análisis y clasificación de los estudios sobre desarrollo social. Infancia y Aprendizaje, 67-68, 163-185.

LOPNNA (2007) Ley Orgánica para la Protección de Niños, Niñas y Adolescentes.

Michelson, L., Sugai, D. P.,Wood, R. P., y Kazdin, A. E. (1987). Las habilidades sociales en la infancia. Barcelona: Martínez Roca.

Monjas, M. (1999). Programa de enseñanza de habilidades de interacción social para niños y niñas en edad escolar (PEHIS). Madrid: CEPE. (4 ${ }^{a}$ Edición). (1 ${ }^{\text {a }}$ Ed. en 1993).

Monjas, M. (2000). La timidez en la infancia y en la adolescencia. Madrid: Pirámide.

Pelechano, V. (1996). Habilidades interpersonales. Teoría mínima y programas de intervención. Vol. II. Programas para niños y adolescentes. Valencia: Promolibro.

Rojas Marcos, L. (1996). Las semillas de la violencia, Madrid, Círculo de Lectores.

Sánchez, S. (2007). Análisis de la presencia de los contenidos de la Cultura de Paz en las iniciativas políticas educativas españolas, Publicaciones, 37, pp. 33-54.

Trianes, M. V. (1996). Educación y competencia social. Un programa en el aula. Málaga: Aljibe.

Trianes, M. V., Jiménez. M., y Muñoz, A. (1997). Competencia social: su educación y tratamiento. Madrid: Pirámide.

Tuvilla, J. (2003). Valores mínimos para crear en la escuela espacios de paz. Actas del I Congreso Hispanoamericano de Educación y Cultura de Paz, Universidad de Granada.

Vinyamata, E. (2005). Conflictología: curso de resolución de conflictos, Barcelona, Ariel. 